\title{
The strong-coupling limit of minimal lattice Landau gauge
}

\section{André Sternbeck* and Lorenz von Smekal}

Centre for the Subatomic Structure of Matter (CSSM), School of Chemistry \& Physics,

The University of Adelaide, SA 5005, Australia

E-mail: andre.sternbeck@adelaide.edu.au, lorenz.smekal@adelaide.edu.au

\begin{abstract}
We study the gluon and ghost propagators of lattice Landau gauge in the strong coupling limit $\beta=0$ in pure $S U$ (2) lattice gauge theory to find evidence of the conformal infrared behavior of these propagators as predicted by a variety of functional continuum methods for asymptotically small momenta $q^{2} \ll \Lambda_{\mathrm{QCD}}^{2}$. In the strong-coupling limit, this same behavior is obtained for the larger values of $a^{2} q^{2}$ (in units of the lattice spacing $a$ ), where it is otherwise swamped by the gauge field dynamics. Deviations for $a^{2} q^{2}<1$ are well parametrized by a transverse gluon mass $\propto 1 / a$. Perhaps unexpectedly, these deviations are thus no finite-volume effect but persist in the infinite-volume limit. They furthermore depend on the definition of gauge fields on the lattice, while the asymptotic conformal behaviour does not.
\end{abstract}

The XXVI International Symposium on Lattice Field Theory

July 14-19 2008

Williamsburg, Virginia, USA

\footnotetext{
* Speaker.
} 


\section{Introduction}

The infrared behaviour of the QCD Green's functions contains essential information about the realisation of confinement in the covariant formulation of QCD, especially in Landau gauge [1]. It has been focus of intensive research over the past ten or so years and was studied with a variety of methods. These include different continuum-based functional methods $[2,3,4,5]$ as well as Monte Carlo (MC) simulations. In particular, the former have predicted that, in Landau gauge, the deep-infrared behaviour of the momentum-space gluon and ghost dressing functions, $Z$ and $G$, should be given by [2]

$$
Z\left(p^{2}\right) \sim\left(p^{2} / \Lambda_{\mathrm{QCD}}^{2}\right)^{2 \kappa_{Z}} \quad \text { and } G\left(p^{2}\right) \sim\left(p^{2} / \Lambda_{\mathrm{QCD}}^{2}\right)^{-\kappa_{G}} \quad \text { for } p^{2} \rightarrow 0,
$$

which are both determined by a unique critical infrared exponent $\kappa_{Z}=\kappa_{G} \equiv \kappa \in(0.5,1)$. Under a mild regularity assumption on the ghost-gluon vertex [3], the value of this exponent is furthermore obtained as $[3,4]$

$$
\kappa=(93-\sqrt{1201}) / 98 \approx 0.595 .
$$

The prediction of this so-called conformal infrared behaviour has been challenged by a number lattice simulations. In fact, with the notable exception of pure $\mathrm{SU}(2)$ lattice gauge theory in 2 dimensions [6], the proposed infrared behaviour (1.1), or even its onset, has not conclusively been confirmed in lattice simulations so far. In contrast, numerical evidence is provided that the dressing functions are consistent with a transverse gluon mass, $Z \sim p^{2} / M^{2}$ and $G=$ const., for $p^{2} \rightarrow 0$.

Finite-volume effects were soon identified as the prime suspects responsible for the disagreement between continuum and lattice results [7]. In fact, in order to be able to see an onset of an at least approximate conformal infrared behaviour in a finite volume of extend $L$, a reasonably large range of momenta satisfying

$$
\pi / L \ll p \ll \Lambda_{\mathrm{QCD}}
$$

needs to be accessible, and it was concluded in [7] that lattice sizes of about $15 \mathrm{fm}$ are needed before even an onset of the leading infrared behaviour can be observed. Not only this has triggered a run for the biggest-ever lattice sizes used in simulations (see, e.g., [8]). All those simulations, however, show very little if no tendency to follow the finite-size corrections to the predicted asymptotic behaviour, even though their volumes reached up to about $20 \mathrm{fm}$ in size.

In this study, we focus on the gluon and ghost propagator in the strong coupling limit, $\beta \rightarrow 0$, of pure $S U(2)$ lattice Landau gauge. This unphysical limit, which can be interpreted as the formal limit $\Lambda_{\mathrm{QCD}} \rightarrow \infty$, allows us to assess whether the predicted conformal behaviour can be seen for the larger lattice momenta $p$, after the upper bound in (1.3) has been removed, in a range where the dynamics due to the gauge action would otherwise dominate and cover it up completely. Our complete analysis can be found in [9].

\section{Gluon and ghost dressing functions in the strong coupling limit}

We simulate $\mathrm{SU}(2)$ gauge theory in the strong coupling limit by generating random link configurations $\{U\}$. These are sets of $\mathrm{SU}(2)$ gauge links, $U_{x \mu}=u_{x \mu}^{0} \mathbf{1}+i \sigma^{a} u_{x \mu}^{a}$, equally distributed over $\left(u^{0}, \vec{u}\right)_{x \mu} \in S^{3}$. Those configurations are fixed to the (standard lattice) Landau gauge (SLG) using an over-relaxation algorithm that iteratively minimises the SU(2) gauge functional of SLG, 

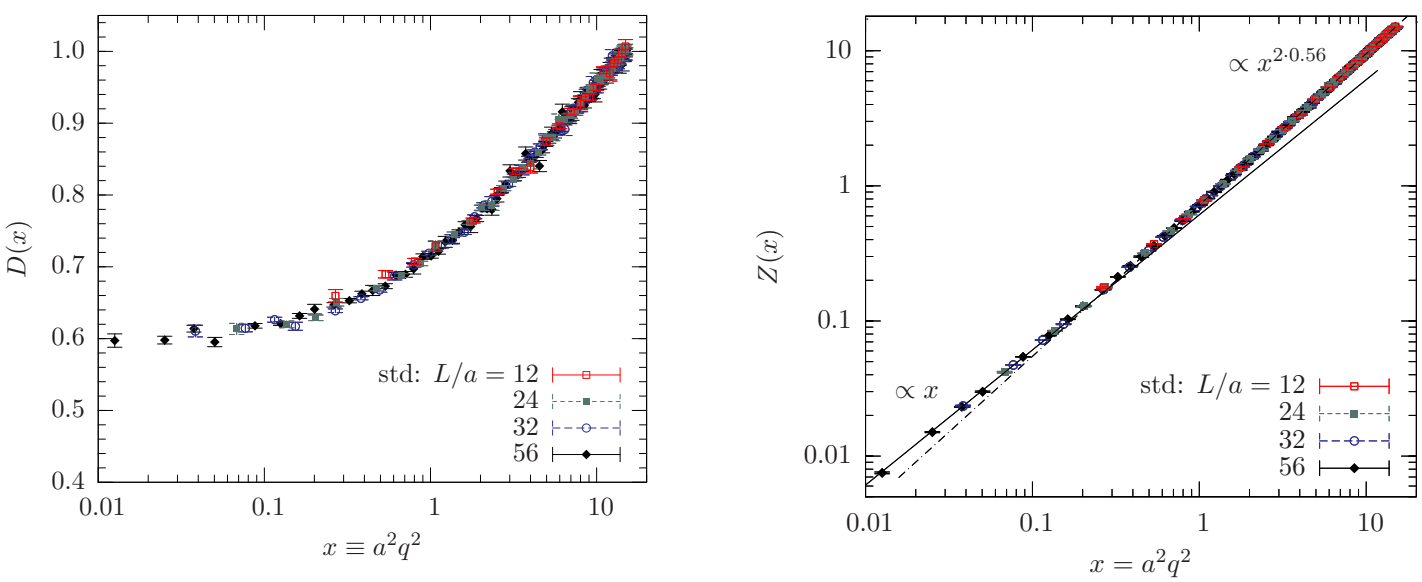

Figure 1: The gluon propagator (left) and its dressing function (right) vs. lattice momentum $a^{2} q^{2}$ for different lattice sizes in the strong coupling limit. Straight lines (right) represent fits of the $56^{4}$ data to its asymptotic behaviour at low (solid) and high (dashed) momenta (see [9]).

$$
V_{U}[g]=4 \sum_{x, \mu}\left(1-\frac{1}{2} \operatorname{Tr} U_{x \mu}^{g}\right) .
$$

To satisfy Landau gauge with sufficient accuracy the over-relaxation algorithm is iterated until the stopping criterion $\max _{x} \operatorname{Tr}\left[\left(\nabla_{\mu}^{b} A_{x \mu}^{g}\right)\left(\nabla_{\mu}^{b} A_{x \mu}^{g \dagger}\right)\right]<10^{-13}$ is met at every lattice site. Here $\nabla_{\mu}^{b}$ denotes the lattice backward derivative and $A_{x \mu}^{g}$ is the lattice gluon field of SLG, given by

$$
A_{x \mu}^{g}=\frac{1}{2 i a}\left(U_{x \mu}^{g}-U_{x \mu}^{g \dagger}\right)
$$

in terms of the gauge-transformed link $U_{x \mu}^{g}$. Gluon and ghost propagators are then calculated in momentum space employing standard techniques. For further details refer to [9].

The data for the gluon propagator and its dressing function is shown in Fig. 1 versus the lattice momentum $a^{2} q^{2}$. The propagator is seen to increase with momentum, while it plateaus at low momenta. Perhaps unexpectedly, however, this happens irrespective of the lattice size at around $a^{2} q^{2} \approx 1$. It is therefore not a finite-volume effect, and the observed mass behaves as $D^{-1}(0) \propto M^{2} \propto 1 / a^{2}$ in the strong-coupling limit with no significant dependence on $L$.

The asymptotic behaviour at either end is also seen in the corresponding dressing function $Z$. We compare this function to fits obtained from the $56^{4}$ data in the right panel of Fig. 1: Writing $x \equiv a^{2} q^{2}$, we use $f(x)=c x^{2 \kappa_{Z}}$ as the fitting function for $x \in[2,14]$ and $f(x)=d x$ for small momenta $x<0.1$ to fit $c, d$ and the exponent $\kappa_{Z}$. The data at large momenta is well described by the power law (1.1) with an exponent $\kappa_{Z}=0.563(1)$, while it approaches the massive behaviour at small $x$.

To assess the model dependence of these results we also used different fit models and a broad range of fitting windows (see [9] for details). Monitoring the corresponding $\chi^{2} /$ ndf values we find that

$$
D_{I}(x)=c x^{2 \kappa_{Z}-1} \quad \text { and } \quad D_{I I}(x)=c(d+x)^{2 \kappa_{Z}-1}
$$

provide stable fits with similar values for $\kappa_{Z}$ and very little dependence on the lattice size. While $D_{I}(x)$ performs slightly better at large momenta, $D_{I I}(x)$ provides good stable fits of the gluon propagator over the whole momentum range. 
The $\kappa_{Z}$ values extracted from different fit models and lattice sizes with fixed window $x \in[2,14]$ are shown in Fig. 2. The observed dependencies on either one are rather small. There is a general trend for $\kappa_{Z}$ to slightly increase with $a / L$ (the dark grey band in Fig. 2) though this is within the systematic uncertainty due to the fit model.

Similar fits were performed to extract the exponent $\kappa_{G}$ from the ghost dressing function $G$. These fits are less robust with a more pronounced model dependence (light grey band in Fig. 2). This is mainly due to the wider transi-

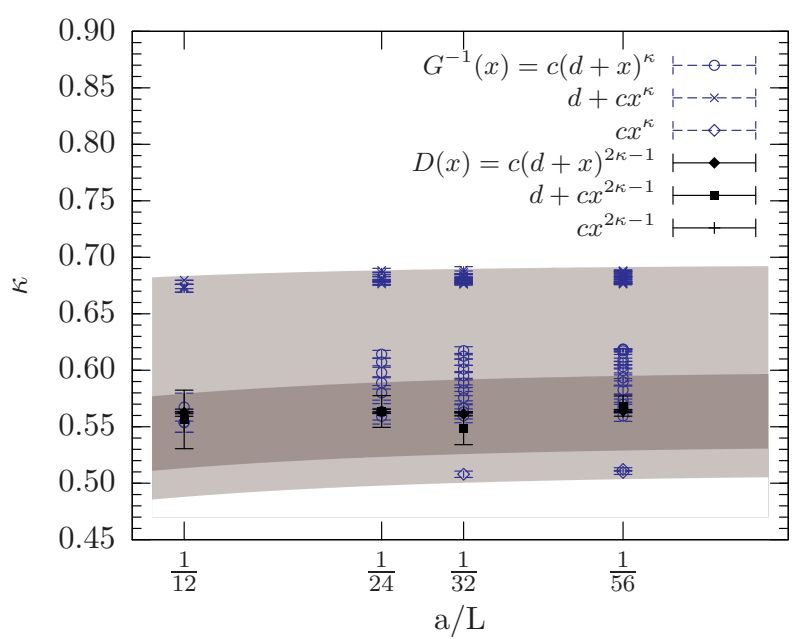

Figure 2: $\kappa$ versus $a / L$ for the gluon and ghost propagators. tion region, from $G=$ const. at small $x$ to $G \sim x^{-\kappa_{G}}$ at large $x$, which is under less control here. The exponent can nevertheless be estimated as $\kappa_{G}=0.60(7)$. The results are consistent with the scaling relation $\kappa_{Z}=\kappa_{G}$.

\section{Comparing different lattice definitions of gauge fields}

It is interesting to compare the above presented SLG data to that of the modified lattice Landau gauge (MLG) introduced in [10] which is based on stereographic projection to define lattice gauge fields. When comparing MLG to the ever popular SLG, there is no advantage that the SLG has over the MLG. A promising particular feature of the MLG on the other hand is that it provides a way to perform gauge-fixed MC simulations sampling all Gribov copies of either sign (of the FaddeevPopov determinant) in the spirit of BRST. This feature will be explored in a forthcoming study. Here we simply use the MLG for comparison in the standard way, i.e., we gauge-fix configurations via minimisation of the MLG functional, which in $\mathrm{SU}(2)$ is of the form [10]

$$
\widetilde{V}_{U}[g]=-8 \sum_{x, \mu} \ln \left(\frac{1}{2}+\frac{1}{4} \operatorname{Tr} U_{x, \hat{\mu}}^{g}\right) .
$$

The MLG functional differs from that of SLG (2.1) and so do the lattice gluon fields of MLG,

$$
\widetilde{A}_{x \mu}=\frac{1}{2 i a}\left(\widetilde{U}_{x \mu}-\widetilde{U}_{x \mu}^{\dagger}\right) \quad \text { where } \quad \widetilde{U}_{x \mu} \equiv \frac{2 U_{x \mu}}{1+\frac{1}{2} \operatorname{Tr} U_{x \mu}} .
$$

The lattice Landau gauge condition $F_{x}=\nabla_{\mu}^{b} A_{x \mu}=0$ is formally unchanged in the stereographically projected gauge fields $\widetilde{A}$, in particular, we have $\widetilde{F}_{x}(A)=F_{x}(\widetilde{A})$. The corresponding Faddeev-Popov operator is given explicitly in [10]. Both lattice definitions of Landau gauge have the same continuum limit, and any differences between MLG and SLG data at finite lattice spacings are lattice artifacts. It is also worth mentioning that gauge configurations fixed to MLG do not satisfy the gauge condition of SLG and vice versa. Nonetheless, exact transversality, i.e., $q_{\mu}(k) A_{\mu}(k)=0$, is 
satisfied at finite lattice spacing $a$ for both of them equally, if the momenta $q_{\mu}(k)$ are defined as $a q_{\mu}(k):=2 \sin \left(\pi k_{\mu} / N_{\mu}\right)$ with integer valued $k_{\mu} \in\left(-N_{\mu} / 2, N_{\mu} / 2\right]$ in the usual way.

The data for the gluon propagator of SLG (red filled diamonds) is compared to that of MLG (blue filled circles) in Fig. 3. There we also show data for the gluon propagator where either

$$
a A_{x \mu}^{\mathrm{adj}}=u_{x \mu}^{0} u_{x \mu}^{a} \sigma^{a}, \quad \text { or } \quad a A_{x \mu}^{\ln }=\phi_{x \mu}^{a} \sigma^{a} / 2 \quad \text { from } U_{x \mu}=\exp \left\{i \phi_{x \mu}^{a} \sigma^{a} / 2\right\}
$$

are used to define lattice gluon fields based on the adjoint representation, $A^{\text {adj }}$ (black open diamonds), and thus blind to the centre [11], or on the tangent space at the identity $A^{\ln }$ (green crosses). In these two cases, $A^{\text {adj }}$ and $A^{\ln }$, for the purpose of a qualitative comparison, we simply use the gauge configurations of the SLG to calculate the gluon propagator. Especially for $A^{\ln }$ this implies, however, that the condition $q_{\mu}(k) A_{\mu}(k)=0$ is satisfied at best approximately and nowhere near the precision of the other two (SLG and MLG). The residual uncertainty due to other possible tensor structures then causes the somewhat larger errors for this definition as seen in Fig. 3.

First, we fit the data from all four definitions to $D_{I I}(x)$ in (2.3) which provides the best overall description in the full momentum range. In order to demonstrate how the other definitions compare to the SLG, we keep its value for the exponent fixed when fitting the other data, i.e., $\kappa_{Z}=0.562$ as obtained for $L / a=32$ in SLG is used in all fits. Relative to the scaling branch $\propto x^{2 \kappa_{Z}-1}$ for large $x=a^{2} q^{2}$ we then observe a strong definition dependence in the (transverse) gluon mass term at small $x$ (see the left panel in Fig. 3). The relative weight of the two asymptotic branches, scaling at large $x$ and massive at small, is clearly discretisation dependent and can not be compensated by finite renormalisations. A first indication that the massive branch might indeed be the ambiguous one is the observed $M \propto 1 / a$. This is consistent with the fact that the definitions of gauge fields on the lattice, which agree at leading order, all differ at order $a^{2}$, and so do their corresponding Jacobian factors which leads to lattice mass counter-terms of different strengths. The observation
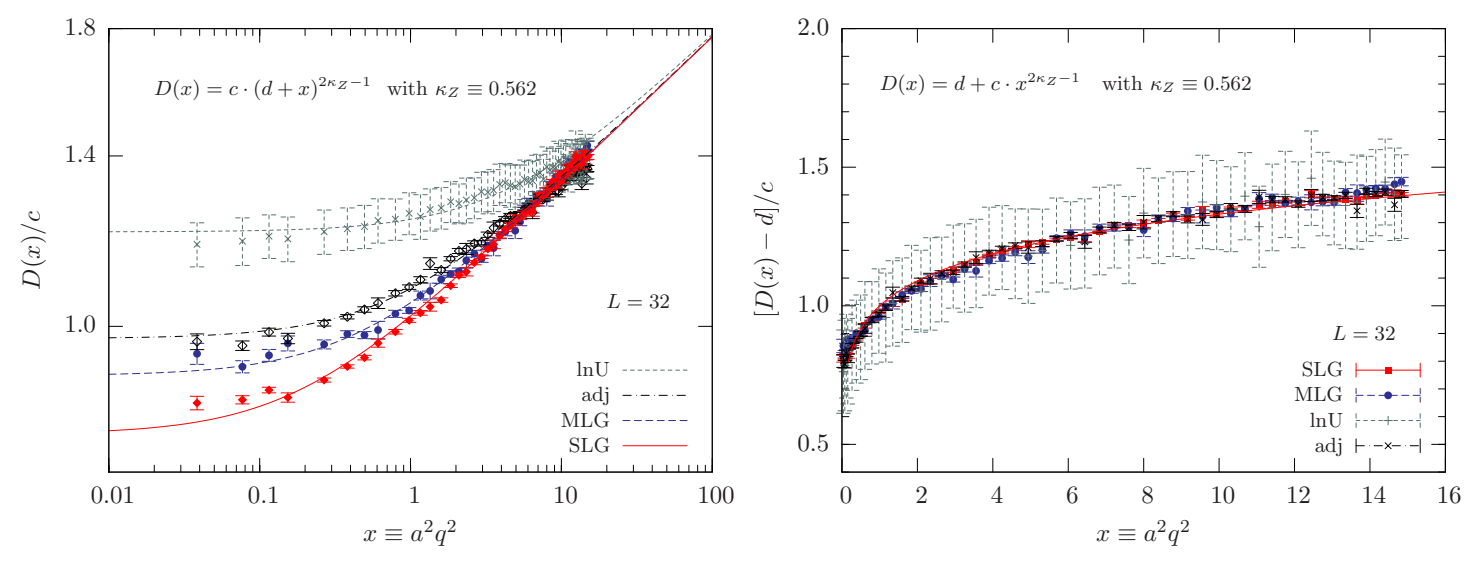

Figure 3: The gluon propagator in the strong-coupling limit over $x=a^{2} q^{2}$ for the various definitions of gauge fields mentioned in the text. All data from $32^{4}$ lattices and normalised to the scaling branch. All fits were performed with fixed $\kappa_{Z}=0.562$ as obtained for the SLG (with $L / a=32$ ). On the left, the results are shown for $D_{I I}(x)$ in (2.3). On the right we use $D_{I I I}=d+c x^{2 \kappa_{Z}-1}$ to demonstrate how the data from all definitions collapse to a unique curve $\propto x^{2 \kappa_{Z}-1}$ (solid, red) when the mass term is subtracted. 
that these differences matter here explicitly demonstrates the breakdown of the lattice SlavnovTaylor identities in minimal lattice Landau gauge in the non-perturbative domain.

To assess whether this ambiguity has an influence on the exponent $\kappa_{Z}$, we have also used fits of the form $D_{I I I}=d+c x^{2 \kappa_{Z}-1}$, again with the fixed $\kappa_{Z} \equiv 0.562$ from the $32^{4}$ SLG data. This form leads to somewhat larger $\chi^{2} / \mathrm{ndf}$ that arise from the transition region around $x=a^{2} q^{2} \sim 1$ which is described a bit better by $D_{I I}$. If we then subtract the constant $d$, however, the normalised data of all four definitions nicely collapse onto a unique curve $\propto x^{2 \kappa_{Z}-1}$ as seen in the right panel of Fig. 3. The data is fully consistent with a unique exponent $\kappa_{Z}$ of around the SLG value which has an infinite volume extrapolation of $0.57(3)$.

The strong-coupling ghost propagators of SLG and MLG are presented in [9]. The best global fits are then obtained for the form $G^{-1}(x)=d+c x^{\kappa_{G}}$. They are again consistent with a unique scaling exponent $\kappa_{G} \approx \kappa_{\mathrm{Z}}$ and deviate from one another in the relative strength of the constant term at small $x=a^{2} q^{2}$. Instead of discussing the strong-coupling ghost propagator in more detail here we turn directly to the product of dressing functions defining a running coupling.

\section{Coupling constant}

The predicted infrared scaling (1.1) with $\kappa_{Z}=\kappa_{G}$ immediately implies that the running coupling defined by [2]

$$
\alpha_{s}\left(p^{2}\right)=\frac{g^{2}}{4 \pi} Z\left(p^{2}\right) G^{2}\left(p^{2}\right)
$$

approaches an infrared fixed point, $\alpha_{s} \rightarrow$ $\alpha_{c}$ for $p^{2} \rightarrow 0$. Standard continuum conventions of course need rescaling $g^{2} Z \rightarrow Z$ when comparing to lattice definitions such as (2.2). The predicted conformal scaling in the strong-coupling limit, with $Z=$ $c_{Z}\left(a^{2} q^{2}\right)^{2 \kappa}$ and $G^{-1}=c_{G}\left(a^{2} q^{2}\right)^{\kappa}$, would therefore imply that the coupling (4.1) is indeed constant with $\alpha_{s}=\alpha_{c}=c_{Z} /\left(4 \pi c_{G}^{2}\right)$. Note that its value is thus determined precisely by those multiplicative constants in the propagators that have been ignored in the analysis up to here. They have to be extracted from the bare lattice data without rescaling or renormalisation. In complete agreement with the general observa-

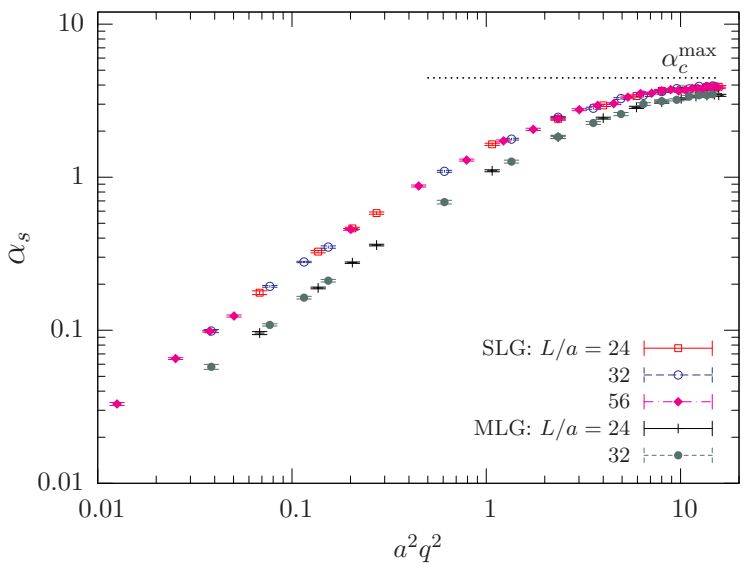

Figure 4: The strong-coupling limit of $\alpha_{s}$ from (4.1) in minimal lattice Landau gauge for SLG and MLG. The dotted line marks $\alpha_{c}^{\max } \approx 4.46$, the maximal value of the critical coupling for $S U$ (2) from the continuum prediction [3]. tion of conformal scaling in the strongcoupling limit at large momenta the product (4.1) of the gluon and ghost dressing functions levels at $\alpha_{c} \approx 4$ for large $a^{2} q^{2}$. As expected for an exponent $\kappa$ slightly smaller than the value in (1.2) this is just below the upper bound $\alpha_{c}^{\max } \approx 4.46$ for $S U(2)$, see [3]. It is quite compelling that also this result is nearly independent of the gauge-field definition, i.e., almost identical for SLG and MLG, see Fig. 4. Predominantly driven by the ghost propagator, the violations to this conformal infrared scaling set in as soon as the ambiguity in the definition of minimal lattice Landau gauge does. 


\section{Conclusions}

We have studied gluon and ghost propagators of pure SU(2) minimal lattice Landau gauge in the strong coupling limit. This unphysical limit probes the gauge field measure of the minimal lattice Landau gauge for there is no contribution from the Yang-Mills (plaquette) action.

As expected for a formal limit $\Lambda_{\mathrm{QCD}} \rightarrow \infty$, it is then observed that the propagators show a conformal scaling behaviour (1.1) for large lattice momenta, $a^{2} q^{2} \gg 1$. Finite-size effects are small and the combined gluon and ghost data is consistent with an $L / a \rightarrow \infty$ extrapolation of a critical exponent $\kappa=0.57(3)$. This scaling branch at large $a^{2} q^{2}$ furthermore leads to a critical coupling of $\alpha_{c} \approx 4$ which is just below the predicted maximum $\alpha_{c}^{\max } \approx 4.46$ for $S U(2)$. These results show very little if no significant dependence on the lattice definition of gauge fields and measure.

Another unambiguous result is the emergence of a transverse gluon mass $M \propto 1 / a$ in the strong-coupling limit of minimal lattice Landau gauge. Both propagators show this massive behaviour at small momenta corresponding to $Z \sim q^{2} / M^{2}$ and $G=$ const. for $a^{2} q^{2} \ll 1$. This massive low momentum branch of the data, however, depends strongly on which lattice definition is being used for the gauge fields and their measure. This is typical for a mass counter-term on the lattice and demonstrates the breakdown of lattice Slavnov-Taylor identities (STIs) and BRST symmetry in minimal lattice Landau gauge beyond perturbation theory. It is still possible that this ambiguity disappears in the continuum limit, eventually. But because it is a combination of ultraviolet (mass counter-term) and infrared (breakdown of STIs) effects, this might take very fine lattice spacings in combination with very large volumes and therefore who-knows-how big lattices to verify explicitly.

This research was supported by the Australian Research Council and by eResearch South Australia.

\section{References}

[1] R. Alkofer and L. von Smekal, Phys. Rept. 353 (2001) 281, [hep-ph / 0007355 ].

[2] L. von Smekal, R. Alkofer, and A. Hauck, Phys. Rev. Lett. 79 (1997) 3591-3594, [hep-ph/9705242]; Ann. Phys. 267 (1998) 1, [hep-ph/9707327].

[3] C. Lerche and L. von Smekal, Phys. Rev. D65 (2002) 125006, [hep-ph/ 0202194 ].

[4] D. Zwanziger, Phys. Rev. D65 (2002) 094039, [hep-th/ 0109224 ].

[5] J. M. Pawlowski, D. F. Litim, S. Nedelko, and L. von Smekal, Phys. Rev. Lett. 93 (2004) 152002, [hep-th/0312324].

[6] A. Maas, Phys. Rev. D75 (2007) 116004, [0 704.0722$].$

[7] C. S. Fischer, A. Maas, J. M. Pawlowski, and L. von Smekal, Annals Phys. 322 (2007) 2916-2944, [hep-ph/0701050].

[8] A. Sternbeck, L. von Smekal, D. B. Leinweber, and A. G. Williams, PoS LAT2007 (2007) 340, [0710.1982]; I. L. Bogolubsky, E. M. Ilgenfritz, M. Müller-Preussker, and A. Sternbeck, PoS LAT2007 (2007) 290, [0 710 . 1968]; A. Cucchieri and T. Mendes, PoS LAT2007 (2007) 297, [0710.0412]; A. Cucchieri and T. Mendes, Phys. Rev. Lett. 100 (2008) 241601, [0712. 3517].

[9] A. Sternbeck and L. von Smekal, in preparation (2008).

[10] L. von Smekal, D. Mehta, A. Sternbeck, and A. G. Williams, PoS LAT2007 (2007) 382, [arXiv:0710.2410 [hep-lat]].

[11] K. Langfeld, H. Reinhardt, and J. Gattnar, Nucl. Phys. B621 (2002) 131-156, [hep-ph/0107141]. 